\title{
Four-Body Scattering of Light as a Method of Detection of Bose-Einstein Condensate of Excitons
}

\author{
Yuri D. Zavorotnev ${ }^{1}$, Vladimir V. Rumyantsev ${ }^{1,2}$, Olga Yu. Popova ${ }^{3}$ \\ ${ }^{1}$ Department of Theory of Complex Systems Dynamic Properties, Galkin Institute for Physics \& Engineering, Donetsk, Ukraine \\ ${ }^{2}$ Mediterranean Institute of Fundamental Physics, Marino, Rome, Italy \\ ${ }^{3}$ International Economics and Marketing Department, Donetsk National Technical University, Pokrovsk, Ukraine
}

\section{Email address:}

zavorotnev.yurii@mail.ru (Y. D. Zavorotnev), vladimir.rumyantsev2011@yandex.ru (V. V. Rumyantsev), olha.popova@donntu.edu.ua (O. Yu. Popova)

\section{To cite this article:}

Yuri D. Zavorotnev, Vladimir V. Rumyantsev, Olga Yu. Popova. Four-Body Scattering of Light as a Method of Detection of Bose-Einstein Condensate of Excitons. Journal of Photonic Materials and Technology. Vol. 4, No. 1, 2018, pp. 39-48.

doi: 10.11648/j.jmpt.20180401.17

Received: April 16, 2018; Accepted: May 3, 2018; Published: May 31, 2018

\begin{abstract}
Process of four-particle light scattering in molecular crystals with participation of the exciton Bose-condensate is considered. The intensities and tensor of scattering are found for this effect. The frequency and polarization characteristics of this process are studies. It is shown that the investigation of the spectrum shapes of scattered radiation allows one to find and prove the existence of the Bose-Einstein condensate of excitons.
\end{abstract}

Keywords: Molecular Crystal, Four-Particle Light Scattering, Exciton, Polaritons, Bose-Einstein Condensate

\section{Introduction}

Effect of condensation of Bose particles in pulse space was predicted by Einstein in 1924-1925 [1, 2]. For the first time, the effect was experimentally detected in 1995 [3-5] in atomic Bose gases. As a result of this outstanding experiment, a branch emerged that dealt with studying of Bose-Einstein condensation (BEC) in gases. As a consequence, interesting effects were found: stimulated reinforcement ("atomic laser"), vortex structures [3-5] etc. The existence of three types of the systems associated with possible BEC was predicted [6]. The first type included the systems where BEC resulted in macroscopic filling of the lowest single energy state. The systems of the second type were characterized by discrete continuum and a finite number of the lowest states. In this case, BEC proceeds in two stages. First, at some temperature, all the states of the system were filled. At the further temperature drop, BEC took place in the lowest state. The third type of the systems was also characterized by two-stage BEC. At the beginning, BEC occurred in the lowest continuum with the infinite number of states that were characterized by macroscopic filling. Studying of BEC gases was complicated because very low temperatures had to be reached $(\sim 100 \mathrm{nK})$, being determined by a relatively large weight of gas particles.

This demerit can be compensated by the study of BEC of other Bose particles, e.g. excitons. The mass of quasiparticles is four-order smaller, so BEC can be registered at much higher temperature. Powerful sources of coherent radiation provide a possibility of formation of the exciton density about $10^{17}-10^{18} \mathrm{~cm}^{-3}$ that makes the collective properties substantial. As exciton excitations of a crystal can be treated as bosons with a reasonable degree of accuracy, Bose-Einstein condensation (BEC) should be detected at low temperatures and repulsive excition-exciton interaction when the values of the wave-vector $\vec{k}$ correspond to the energy minimum [7]. Later a number of papers was published that were aimed at detection and testing of BEC excitons. In the pioneer works, emergence of a narrow luminescence peak was the main evidence of detection of the condensate. But the application of this method to three-dimensional crystals did not resulted in reliable identification of BEC because of short lifetime of excitons. This fact did not allow accumulation of sufficiently large amount of quasi-particles in the lowest state. The main factors reducing the lifetime were various non-radiative relaxation processes. In order to extend the lifetime of quasiparticles, attempts of forming 
BEC within quantum confinements or in composite sandwich-like materials were made. For most experiments to date, exciton-polariton condensates are produced at cryogenic temperatures in the vicinity of $\sim 10 \mathrm{~K}$ using materials such as GaAs and CdTe. However, using other materials such as $\mathrm{GaN}, \mathrm{ZnO}$, and organic semiconductors, polariton condensates at higher temperatures including room temperature have been also realized [8-11]. Theoretical works dealing with the problem of detection of exciton BEC were mostly concerned with the spectrum calculations and the following conclusion about the position of the luminescence line and the shape of the tails [12, 13]. In [12], incorrect application of Belyaev method was made [14], that was valid only in the case when the condensate was the ground state of the system and BEC of excitons was an excited state. The list of possible candidates able to demonstrate BEC was recently supplemented with a new type of quasiparticles [15]. In particular, it was shown [16] that in the case of a two-dimensional system of spin-less electrons under quantum Hall effect, so-called composite particles (CP) could emerge. These objects are point vortices fixed to a bare particle. At certain conditions, CPs obey Bose statistics and BEC can be realized. Review [17] was focused upon several physical phenomena exhibited by excitonpolariton condensates. In particular, the topics related to the difference between a polariton BEC, a polariton laser, and a photon laser, as well as physical phenomena such as superfluidity, vortex formation, BKT (Berezinskii-KosterlitzThouless) and BCS (Bardeen-Cooper-Schrieffer) physics were examined. The physics and application of engineered polariton structures was also discussed. The results of studying of BEC excitons form the basis of application of the effect. In particular, a possibility of the existence of an exciton dielectric [18] and the related double-wire superconducting line [19] is predicted

Having regard to the above, the existence proof of BEC requires additional methods beside luminescence. The related opportunity exists because BEC of excitons interacts with other subsystems, e.g. phonon and photon ones. As a result, the spectrum of the elementary excitations is modified. It is demonstrated in [15] that the presence of BEC excitons makes possible the appearance of additional polariton states. In [20], acoustic methods of testing are suggested to be applied. In particular, it is shown that unusual behavior of the absorption coefficient of surface acoustic waves is determined by BEC exciton gas. Besides, various non-linear optical effects can be used [21-23]. They provide BEC detection at the state of $k=0$. These states cannot irradiate because the use of luminescence implies $k \neq 0$. But an exciton can be drawn out of this state by means of various non-linear processes. In particular, in [23, 24], a method of double-photon recombination is developed. The main idea is radiation of two excitons characterized by the opposite impulses of the same moduli. In the present work, one on numerous non-linear processes is suggested to be applied to the testing and identification of BEC excitons. BEC excitons associated with both zero and non-zero impulse can participate in the process.

\section{Theoretical Background}

Consider a cubic molecular crystal with one molecule in the unit cell. Assume that a Bose - Einstein condensate (BEC) of excitons has been created in the crystal on branch $\sigma$ for $k=0$ and at the temperature of $0 K$. The energy per one BEC quasiparticle [20] is equal to the chemical potential $\mu$.

Let us investigate a process of nonlinear scattering when a polariton of energy $\hbar \omega_{1} \equiv E_{\rho_{1}}\left(\vec{k}_{1}\right)$ and a condensate exciton are annihilated, while a scattered polariton $\hbar \omega_{2} \equiv E_{\rho_{2}}\left(\vec{k}_{2}\right)$ and a supracondensate exciton are born at branch $v$. The process is represented in Figure 1, where the wavy lines stand for polaritons, while the dotted and solid lines correspond to the condensate and supracondensate excitons.

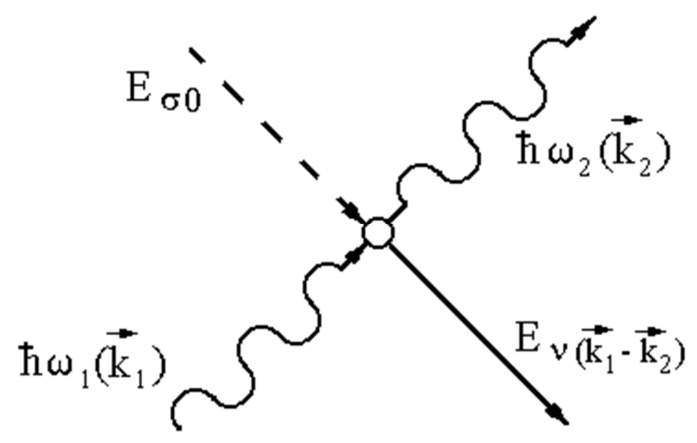

Figure 1. The scheme of the four-body process. The wavy lines stand for polaritons, while the dotted and solid lines correspond to the condensate and supracondensate excitons.

The problem could be solved within the framework of exciton - polariton Hamiltonian which takes into account the summands of the third and fourth order terms in operators of quasi - particle generation and annihilation. However, the use of interaction constants shown in $[25,26]$ gives a very bulky scattering tensor. To simplify the tensor, the method of acting field was applied [27, 28].

As known [29], in molecular crystals, the electronic molecular escitations obey the Pauli statistics. But after the transition from the Pauli operators to Bose operators with using the procedure developed in [30], the Hamiltonian of the system composed by the crystal and the field of transverse photons can be represented as

$$
\begin{aligned}
H= & \frac{1}{2} \sum\left\{\left(\vec{p}_{n f}-\sqrt{\frac{v_{0}}{4 \pi}} \frac{\omega_{0}}{c} \vec{A}_{n}\right)^{2}+\omega_{f}^{2} q_{n f}^{2}\right\}+V_{c}+ \\
& +\int d V\left(\frac{E^{2}+H^{2}}{8 \pi}\right),
\end{aligned}
$$

where $\vec{p}_{n f}, \vec{q}_{n f}$ are generalized momentum and the coordinate of molecular cell of number $n$, which are expressed through Bose operators 


$$
\begin{aligned}
& \vec{q}_{n f}=\sqrt{\frac{\hbar}{2 m \omega_{f}}}\left(b_{n}+b_{n}^{+}\right) \vec{e}_{n f}, \\
& \vec{p}_{n f}=i \sqrt{\frac{m \hbar \omega_{f}}{2}}\left(b_{n}^{+}-b_{n}\right) \vec{e}_{n f},
\end{aligned}
$$

here $\vec{e}_{n f}$ is the unit vector; $v_{0}$ is the volume of a crystal unit cell; $\omega_{0}$ is the plasma frequency, $\omega_{f}$ is the oscillation frequency of number $f ; V_{c}$ is the Coulomb interaction between the molecules in the dipole - dipole approximation [28]; $\vec{A}_{n}$ is the vector potential at site n; $\vec{E}, \vec{H}$ are the densities of electrical and magnetic field of the external electromagnetic wave, respectively. It should be noted that in the Hamiltonian (1), the kinematic interaction [29] is not taken into consideration due to non - Bose elementary excitations. Such an approximation is justified for the case of four - particle processes only for specially taken resonance conditions that will be discussed further. If (1) is subjected to canonical transformation of the form [31]

$$
H^{\prime}=U H U^{-1}
$$

where

$$
U=\exp \left(\frac{i}{\hbar c} \sum_{n} \vec{d}_{n} \vec{A}_{n}\right)
$$

and

$$
\vec{d}_{n}=\omega_{0} \sqrt{\frac{v_{0}}{4 \pi}} \sum_{f} \vec{q}_{n f}
$$

is the dipole moment of site $n$, the Hamiltonian of a cubic crystal can be represented as [32]

$$
\begin{aligned}
& H=\frac{1}{2} \sum_{n f}\left(\tilde{p}_{n f}^{2}+\omega_{f}^{2} q_{n f}^{2}\right)+ \\
& +\int d V\left\{\frac{\left(\vec{D}-4 \pi \overrightarrow{\mathrm{P}}_{L}\right)^{2}+\vec{H}^{2}}{8 \pi}-\frac{2 \pi}{3} \mathrm{P}_{L}^{2}\right\}-\sum_{n} \vec{d}_{n}^{N L} \vec{E}_{n}^{e f} .
\end{aligned}
$$

In (5) the following designations are introduced:

$$
\tilde{\vec{p}}_{n f}=\vec{p}_{n f}-\frac{\omega_{0}}{c} \sqrt{\frac{v_{0}}{4 \pi}} \vec{A}_{n}
$$

is a new generalized momentum; $\vec{D}$ is the vector of electrical induction; $\overrightarrow{\mathrm{P}}_{L}$ is the linear part of crystal polarization shown by the expression

$$
\overrightarrow{\mathrm{P}}_{L}(\vec{r})=\sum_{n} \vec{d}_{n} \delta\left(\vec{r}-\vec{r}_{n}\right)
$$

$\vec{d}_{n}^{N L}$ is the nonlinear part of the dipole moment);

$$
E_{n i}^{e f}=E_{n i}-\frac{4 \pi}{v_{0}} \sum_{m} Q_{n m}^{i j} d_{m}^{j}
$$

is a field acting on the molecule, $\vec{E}_{n}$ is the external field density at site $n$;

$$
\begin{aligned}
& Q_{n m}^{i j}=N^{-1} \sum_{\vec{k}} \exp \left[i \vec{k}\left(\vec{r}_{n}-\vec{r}_{m}\right)\right] \times \\
& \times\left\{\frac{1}{3} \delta_{i j}-\sum_{\vec{g}}\left[\frac{(\vec{k}+\vec{g})_{i}(\vec{k}+\vec{g})_{j}}{(\vec{k}+\vec{g})^{2}}-\frac{1}{3} \delta_{i j}\right]\right\}
\end{aligned}
$$

is the coefficient of the internal field [28]; indices $i, j$ run independent of $x, y, z$ values; the summation over $\vec{k}$ and $\vec{g}$ in (9) is done for the first Brillouin zone and in other zones, respectively.

Within the secondary quantization, the first two summands of expression (5) are reduced to diagonal type by transition to the states of mechanical excitons [29] and then to polaritons. In this case, the last summand of (5), which in the approximation of secondary quantization is cubic over the operators of quasi - particles, takes the form

$$
\begin{aligned}
& H^{I I I}=N^{-\frac{3}{2}} \sum_{\substack{\vec{k}_{1} \vec{k}_{2} \vec{k}_{3} \\
\alpha_{1} \alpha_{2} \alpha_{3}}}\left\{R_{\alpha_{1} \alpha_{2} \alpha_{3}}^{(1)}\left(\vec{k}_{1}, \vec{k}_{2}, \vec{k}_{3}\right) A_{\alpha_{1} \vec{k}_{1}}^{+} A_{\alpha_{2} \vec{k}_{2}} A_{\alpha_{3} \vec{k}_{3}}+\right. \\
& \left.+R_{\alpha_{1} \alpha_{2} \alpha_{3}}^{(2)}\left(\vec{k}_{1}, \vec{k}_{2}, \vec{k}_{3}\right) A_{\alpha_{1} \vec{k}_{1}} A_{\alpha_{2} \vec{k}_{2}} A_{\alpha_{3} \vec{k}_{3}}+\text { э.c. }\right\}
\end{aligned}
$$

The indices of $\alpha$ run all values corresponding to branches of polaritons $A_{\vec{k} \rho}$ and mechanical excitons $A_{\vec{k} s}$. Later on, from the whole set of the constants $R^{(1)}$ and $R^{(2)}$, only $R_{s_{1} s_{2} \rho}^{(1)}, R_{\rho_{1} \rho_{2} s_{1}}^{(1)}$ and $R_{s_{1} \rho_{1} \rho_{2}}^{(1)}$ are required, which are written down in the following form:

$$
\begin{aligned}
& R_{s_{1} s_{2} \rho}^{(1)}\left(\vec{k}_{1}, \vec{k}_{2}, \vec{k}_{3}\right)=\left(4 \pi v_{0}\right)^{3} \sum_{i n} e^{i\left(\vec{k}_{2}+\vec{k}_{3}-\vec{k}_{1}\right) \vec{r}_{n}} d_{s_{1} S_{2}}^{i} \varepsilon_{\vec{k}_{3} \rho}^{i}, \\
& R_{\rho_{1} \rho_{2} s}^{(1)}\left(\vec{k}_{1}, \vec{k}_{2}, \vec{k}_{3}\right)=\left(4 \pi v_{0}\right)^{3} \hbar^{-1} \sum_{i n s_{1} j} e^{i\left(\vec{k}_{2}+\vec{k}_{3}-\vec{k}_{1}\right) \vec{r}_{n}} d_{0 s_{1}}^{j} d_{s_{1} s}^{i} * \\
& *\left[\frac{\varepsilon_{\vec{k}_{1} \rho_{1}}^{i} \varepsilon_{\vec{k}_{2} \rho_{2}}^{j}}{\omega_{s_{1}}-\omega_{\vec{k}_{2} \rho_{2}}}+\frac{\varepsilon_{\vec{k}_{2} \rho_{2}}^{i} \varepsilon_{\vec{k}_{1} \rho_{1}}^{j}}{\omega_{s_{1}}+\omega_{\vec{k}_{1} \rho_{1}}}\right], \\
& R_{s \rho_{1} \rho_{2}}^{(1)}\left(\vec{k}_{1}, \vec{k}_{2}, \vec{k}_{3}\right)=\left(4 \pi v_{0}\right)^{3} \hbar^{-1} \sum_{i n s_{1} j} e^{i\left(\vec{k}_{2}+\vec{k}_{3}-\vec{k}_{1}\right) \vec{r}_{n}} d_{s s_{1}}^{i} d_{s_{1} 0}^{j} * \\
& *\left[\frac{\varepsilon_{\vec{k}_{2} \rho_{2}}^{i} \varepsilon_{\vec{k}_{1} \rho_{1}}^{j}}{\omega_{s_{1}}-\omega_{\vec{k}_{2} \rho_{2}}}\right],
\end{aligned}
$$

where $d_{s_{1} s_{2}}$ is a matrix element of the operator of dipole moment over the states of mechanical excitons; $\vec{\varepsilon} \vec{k} \rho$ is the vector of the electric intensity for a polarition wave in the crystal [29]. 
As the effect is a four - body one, then the summands which are cubic over the operators of quasi - particles will contribute to the effect of the second order of the perturbation theory, while the fourth - order summands are associated with in the first-order one.

The process is schematically shown in Figure 2. Here the dotted and the straight lines show the condensate and supracondensate excitons, while the wavy line expresses the polaritons. On the supposition that one of the following resonance conditions is satisfied:

$$
\begin{aligned}
& \text { 1) } E_{s} \sim \hbar \omega_{1}+E_{\sigma 0}, \\
& \text { 2) } E_{\rho}\left(\vec{k}_{1}\right) \sim \hbar \omega_{1}+E_{\sigma 0}, \\
& \text { 3) } \hbar \omega_{2} \sim E_{\sigma 0}-E_{s}, \\
& \text { 4) } \hbar \omega_{2} \sim E_{\sigma 0}-E_{\rho}\left(-\vec{k}_{2}\right)
\end{aligned}
$$

In this case the dominating contribution to nonlinear scattering will be formed by the cubic summands of the Hamiltonian (10) in the second order of the perturbation theory, while the contribution of the fourth - order summands may be neglected, a part of which is determined by the kinematic interaction.

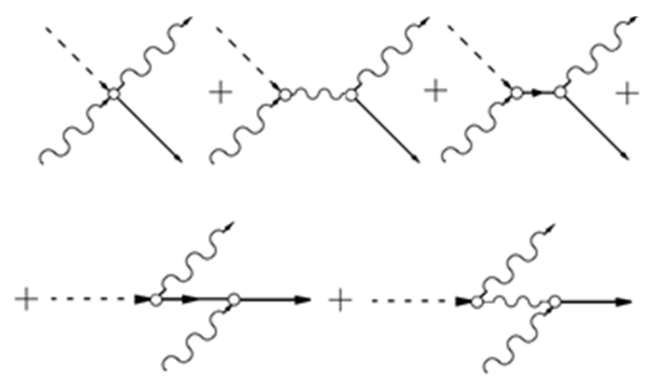

Figure 2. Thescheme of the four-body process sin the first and second order of perturbation theory. The wavy lines stand for polaritons, while the dotted and solid lines correspond to the condensate and supracondensate excitons.

Now the energy flow of scattered radiation $S$ should be found. Two different situations are considered.

1) Let in the final state, alongside with the polariton, a mechanical exciton on branch $v$ emerges. The energy of the exciton does not depend on the wave vector [28]. Then, for the energy flow of the scattered radiation to the unit solid angle $d \Omega$, the following expression is written:

$$
\frac{\partial S}{\partial \Omega}=I V n_{0} A\left(\omega_{1}, \omega_{2}\right) \sum_{n=1}^{l_{\varpi}} \sum_{m=1}^{l_{v}}\left|\sum_{i j} a_{i j}^{n m}\left(\omega_{1}, \omega_{2}\right) \varepsilon_{\vec{k}_{1} \rho_{1}}^{i} \varepsilon_{\vec{k}_{2} \rho_{2}}^{j}\right|^{2}
$$

Where $I$ is the intensity of monochromatic pumping of frequency $\omega_{1} ; n_{0}$ is the exciton density in the condensate; $l_{\sigma}, l_{v}$ is the degeneracy multiplicity for the initial (condensate) and final (supracondnsate) excitonic states, respectively; $\omega_{2}$ is the scattered radiation frequency which satisfies the law of momentum conservation

$$
\hbar \omega_{1}\left(\vec{k}_{1}\right)+E_{\sigma 0}=\hbar \omega_{2}\left(\vec{k}_{2}\right)+E_{v\left(\vec{k}_{1}-\vec{k}_{2}\right)} ;
$$

$$
A\left(\omega_{1}, \omega_{2}\right)=\frac{4 \omega_{2}^{3} v_{0}^{2} n_{2}^{2}}{\hbar^{2} \omega_{1} v_{1} v_{2} c^{2}}
$$

$n_{i}, v_{i}(i=1,2)$ is the index of refraction and group velocity of the wave at frequency $\omega_{i} ; a_{i j}^{n m}$ is the scattering tensor; indices $n$ and $m$ characterize the initial and final states, respectively.

2) The both quasi - particles emerged at the final state are polaritons. Similar to parametric luminescence or generation of the second harmonic [25], the flow of energy of scattered radiation will highly depend on the scattering angle. Thus, the phase synchronism conditions should be taken into consideration.

Let an isotropic crystal be cut out in the form of a parallelsided plate of small thickness along $O Z$ axis. Vector $\vec{k}_{1}-\vec{k}_{2}=\vec{k}_{3}$ is directed along the same axis. Then, the expression for the energy flow of scattered radiation to the unit solid angle $d \Omega$ in the spectral interval from $\omega_{2}$ to $\omega_{2}+d \omega_{2}$ is written as follows

$$
\begin{aligned}
& \frac{\partial^{2} S}{\partial \omega_{2} \partial \Omega}=\frac{I n_{0} L_{x} L_{y} A\left(\omega_{1}, \omega_{2}\right)}{2 \pi v_{\rho_{3}}} \sum_{n=1}^{l_{\sigma}}\left|\sum_{i j} a_{i j}^{n m}\left(\omega_{1}, \omega_{2}\right) \varepsilon_{\vec{k}_{1}}^{i} \varepsilon_{\vec{k}_{2}}^{j}\right| * \\
& * \frac{\sin ^{2}\left(\frac{\Delta k_{z} L_{z}}{2}\right)}{\left(\Delta k_{z}\right)^{2}},
\end{aligned}
$$

where $L_{x}, L_{y}, L_{z}$ are linear dimensions of the crystal along axes $O X, O Y, O Z$, respectively; $v_{\rho_{3}}$ is the group velocity of the polariton associated with wave vector $\vec{k}_{3}$; $\Delta \vec{k}=\vec{k}_{1}-\vec{k}_{2}-\vec{k}_{3}$. For the first resonant situation, tensor $a_{i j}^{n m}$ can be represented as

$$
a_{i j}^{n m}(1)=\sum_{s} \frac{d_{n s}^{i} d_{s m}^{j}}{E_{s}-\hbar \omega_{1}-E_{\sigma 0}} .
$$

For other resonant conditions we have

$$
\begin{gathered}
\left.a_{i j}^{n m}(2)=\frac{4}{\hbar^{2}} \sum_{s_{1} s_{2} l \rho} \frac{d_{n s_{1}}^{i} d_{s_{1} 0}^{l} d_{0 s_{2}}^{l} d_{s_{2} m}^{j}\left(\varepsilon_{\vec{k}_{1} \rho}^{l}\right)^{2}}{E_{s}-\hbar \omega_{1}-E_{\sigma 0}} \frac{\left(\omega_{s_{1}}^{2}+\omega_{k_{1} \rho}^{2} \rho\right)\left(\omega_{s_{2}}^{2}+\omega_{\vec{k}_{1} \rho}^{2} \rho\right.}{\left(\omega_{s_{1}}^{2}-\omega_{k_{1} \rho}^{2}\right)\left(\omega_{s_{2}}^{2}-\omega_{k_{1}}^{2} \rho\right.}\right) \\
a_{i j}^{n m}(3)=\sum_{s} \frac{d_{n s}^{j} d_{s m}^{i}}{E_{s}-E_{\sigma 0}+\hbar \omega_{2}} ; \\
a_{i j}^{n m}(4)=4 \hbar^{-2} \sum_{s_{1} s_{2} \rho l} \frac{d_{n s_{1}}^{j} d_{s_{1} 0}^{l} d_{0 s_{2}}^{l} d_{s_{2} m}^{i}\left(\varepsilon_{-\vec{k}_{2} \rho}^{l}\right)^{2}}{E_{\rho}\left(-\vec{k}_{2}\right)-E_{\sigma 0}+\hbar \omega_{2}} * \\
* \frac{1}{\left(\omega_{s_{1}}-\omega_{-\vec{k}_{2} \rho}\right)\left(\omega_{s_{2}}-\omega_{-\vec{k}_{2} \rho}\right)}
\end{gathered}
$$

Let us investigate the tensor of nonlinear scattering. Now 
the theoretical group analysis of tensor $a_{i j}^{n m}$ is performed. Suppose that there is the first resonant situation described above. Then $a_{i j}^{n m}(1)$ can be represented in the form

$$
a_{i j}^{n m}(1)=<n\left|d^{i} \Lambda\left(\hbar \omega_{1}+E_{\varpi 0}\right) d^{j}\right| m>
$$

where

$$
\Lambda(\hbar \omega)=\sum_{s} \frac{|s><s|}{E_{s}-\hbar \omega} .
$$

In the limit $\vec{k} \rightarrow 0$, operator $\Lambda$ is invariant with respect to the point group of the crystal. This group is a quotient group of the space group of the crystal with respect to the translation subgroup. Thus, it is convenient to represent tensor $a_{i j}^{n m}(1)$ in the form

$$
a_{i j}^{n m}(1) \sim \int \psi_{n}^{\sigma} a_{i j} \psi_{m}^{v} d \tau
$$

where $a_{i j}$ is a tensor of rank two; $\psi_{n}^{\sigma}, \psi_{m}^{v}$ are the $n$-th and $m$-th basic wave functions of representations $\Gamma_{\sigma}$ and $\Gamma_{v}$, respectively. Non-zero expression (25) is realized only if tensor $a_{i j}$ contains a function transformed in the same way as product $\psi_{n}^{\sigma} \psi_{m}^{\nu}$. As the systems of functions $\psi_{1}^{\sigma}, \psi_{2}^{\sigma}, \ldots, \psi_{l_{\sigma}}^{\sigma}$, and $\psi_{1}^{v}, \psi_{2}^{v}, \ldots, \psi_{l_{v}}^{\nu}$, are the basic functions of irreducible representations $\Gamma_{\sigma}$ and $\Gamma_{v}$ respectively, then their products are the basis in the general case of a reducible representation of dimension $l_{\sigma} l_{v}$ These products are irreducible when at least one of $l_{\sigma}$ or $l_{v}$ equals unity. In this case, products of the type $\psi_{n}^{\sigma} \psi_{m}^{v}$ are basic functions of an irreducible representation $\Gamma_{\alpha}$ determined by the relation

$$
\Gamma_{\sigma} \times \Gamma_{v}=\Gamma_{\alpha}
$$

Projecting tensor $a_{i j}$ onto the basic function of representation $\Gamma_{\alpha}$, one can determine nonzero components of the first summand in expression for $a_{i j}^{n m}(1)$.

Now, let both representations $\Gamma_{\sigma}$ and $\Gamma_{v}$ be other than one-dimensional. In this case, the set $\psi_{n}^{\sigma} \psi_{m}^{v}$ should be expanded in basic functions of irreducible representations according to formula [33].

$$
\psi_{n}^{\sigma} \psi_{m}^{v}=\sum_{\alpha, s} \psi_{s}^{\alpha}
$$

where

$$
\psi_{s}^{\alpha}=\frac{l_{\alpha}}{g} \sum_{G} G_{s s}^{(\alpha)^{*}} \hat{G}\left(\psi_{n}^{\sigma} \psi_{m}^{v}\right)
$$

$g$ is a number of group elements; $\hat{G}$ is a symmetry operation; $G_{s s}^{(\alpha)}$ are diagonal elements of transformation matrix. Next, as follows from (25) and (26), it is necessary to project $a_{i j}$ on every function $\psi_{s}^{\alpha}(27)$ and to add the produced matrices together.

Tensor $a_{i j}(3)$ (21) is analyzed in the same way.

Now it is assumed that only the second resonant situation is correct. In this case, tensor $a_{i j}^{n m}(2)$ can be represented as

$$
a_{i j}^{n m}(2) \sim \sum_{l \rho} \frac{<n\left|d^{i} d^{l}\right| 0><0\left|d^{l} d^{j}\right| m>\left(\varepsilon_{\vec{k}_{1} \rho}^{l}\right)^{2}}{E_{\rho}\left(\vec{k}_{1}\right)-\hbar \omega_{1}-E_{\sigma 0}}
$$

As follows from (29), $a_{i j}^{n m}(2)$ is a convolution of two second-rank tensors projected on the irreducible representation $\Gamma_{\sigma}$ and $\Gamma_{v}$, respectively. The appropriate tables are given in [34]. Analysis of $a_{i j}^{n m}(4)$ should be done in the same way.

As an example, the scattering in crystal of symmetry $T_{h}$ is considered. Let $\mid n>$ and $\mid m>$ be transformed as the $X$ - th component of the axial vector. Then, product $|n\rangle|m\rangle$ is also transformed as $|n\rangle$, and tensor $a_{i j}^{n m}$ (1) will have the form [27]

$$
\left(\begin{array}{lll}
0 & 0 & 0 \\
0 & 0 & a \\
0 & a & 0
\end{array}\right)
$$

To establish the type of $a_{i j}^{n m}(3)$, it is necessary to find the square of matrix (30). Thus,

$$
\left(\begin{array}{lll}
0 & 0 & 0 \\
0 & b & 0 \\
0 & 0 & b
\end{array}\right)
$$

As seen from (30) and (31), the form of the scattering tensor is varied under different resonant situations.

\section{Some Corollaries of Laws of Conservation for a Four - Body Process}

As follows from expression (18), in the case when condensate and supracondensate quasi - particles are at the upper polariton branch, the energy flow of scattered radiation will be maximal if the laws of conservation operate simultaneously. 


$$
\left\{\begin{array}{l}
\hbar \omega_{1}\left(\vec{k}_{1}\right)+E_{\sigma 0}=\hbar \omega_{2}\left(\vec{k}_{2}\right)+E_{\sigma \vec{k}_{3}} \\
\vec{k}_{1}=\vec{k}_{2}+\vec{k}_{3}
\end{array}\right.
$$

Taking into account [29] that $\left|\vec{k}_{i}\right|=\frac{\omega_{i}}{c} n_{i}$ (where $n_{i}=n\left(\omega_{i}\right)$ is the index of refraction at the appropriate frequency), from system (32), scattering angle $\theta$ is found, which satisfies the conditions of phase synchronism

$$
\sin \frac{\theta}{2}=\frac{\left(\omega_{1}-\omega_{2}+\omega_{\sigma}\right)^{2} n_{\sigma}^{2}-\left(\omega_{1} n_{1}-\omega_{2} n_{2}\right)^{2}}{4 n_{1} n_{2} \omega_{1} \omega_{2}}
$$

In (33), $n_{\sigma}$ corresponds to frequency $\omega_{\sigma}=\hbar^{-1} E_{\sigma \vec{k}_{3}}$. $E_{\sigma \vec{k}_{3}}>E_{\sigma 0}$ then in direction of the maximal intensity of scattered radiation, the scattering spectrum is similar to that represented in Figure 3, where $I$ is an intensity of scattered radiation; lines of frequencies $\left(\omega_{1}-E_{v} \hbar^{-1}\right)$ and $\omega_{2}$ respond to ordinary combination scattering and condensate - aided scattering, respectively. Moreover, due to the $\delta$ - shaped function of the condensate exciton distribution over the quasi - impulses, there is a coincidence in the spectral forms of incident and scattered radiation, in the case of narrow band pumping.

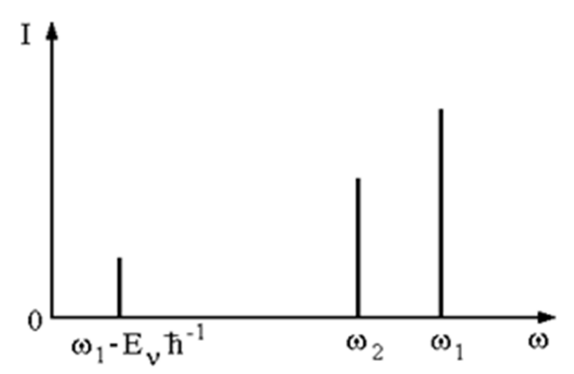

Figure 3. Frequency spectrum of the process. I is the intensity of the scattered radiation; the lines at the frequencies of $\left(\omega_{1}-E_{v} \hbar^{-1}\right)$ and $\omega_{2}$ are associated with conventional combination scattering and scattering with the condensate involved.

Now the case is considered when an exciton originates in the final state. In the crystal, the energy of real excitons depends on the wave vector. Therefore it is necessary to take into account the presence of synchronism and to determine directions of the largest energy transformation by using the system (32). To be definite, it is assumed that the law of exciton dispersion is parabolic, i.e.

$$
E(\vec{q})=E(0)+\frac{\hbar^{2} q^{2}}{2 m},
$$

where $m$ is effective mass. For polaritons, the relation $\omega=|\vec{k}| c n^{-1}$ is still used

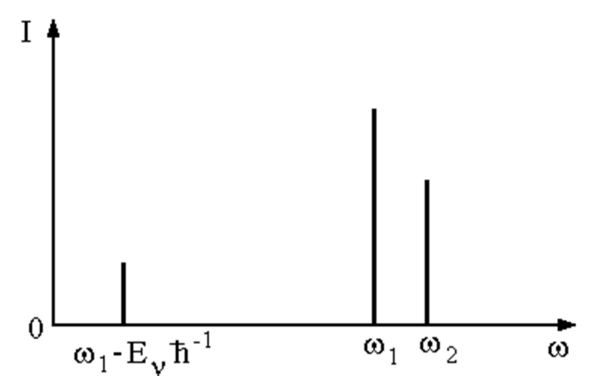

Figure 4. Spectrum in the case of $E_{v \vec{q}}<E_{\sigma 0}$.

Let us study some cases.

1. Condensate and supracondensate excitons belong to different branches $\sigma$ and $v$. According to the law of energy conservation, for $E_{\vec{v}}>E_{\sigma 0}$, the scattering spectrum will look like that of Fiure 3. For $E_{v \vec{q}}<E_{\sigma 0}$, the spectrum is shown in Figure 4.

Similar to the effect of second - harmonic generation [35], the cases of collinear and vector phase synchronisms are probable. Let us first consider the collinear phase synchronism.

1. Forward scattering. In this case, the wave vectors of pumping and of scattered radiation are collinear and unidirectional. Then, by elimination of one equation from system (32) with condition of (34) taken into account, we have

$$
\left[\omega_{1} n_{1}(\theta, \phi)-\omega_{2} n_{2}(\theta, \phi)\right]^{2}=\frac{2 m c^{2}}{\hbar^{2}}\left[\hbar \omega_{1}-\hbar \omega_{2}+E_{\sigma 0}-E_{v 0}\right]
$$

where $\theta$ and $\phi$ are polar angles characterizing the direction of wave vectors. In formula (35), the left - hand part is a function of $\theta$ and $\phi$, while the right -hand one is not. As, in the general case, $n(\theta, \phi)$ is a rather complex surface, it is clear that (35) will be satisfied only in preferred directions of $\vec{k}$ that should be calculated for each crystal with using parameters determining the shape of the surface of indices. In a cubic crystal, the surface $n(\theta, \phi)$ is spherical and equation (35) can only be exceptionally satisfied for specially selected values of $\omega_{1}, \omega_{2}, E_{\sigma 0}$.

2. Back scattering. Wave vectors $\vec{k}_{1}$ and $\vec{k}_{2}$ are collinear as well but their direction is opposite. From (32) after simple transformations, we have

$$
\left[\omega_{1} n_{1}(\theta, \phi)+\omega_{2} n_{2}(\pi-\theta, \pi-\phi)\right]^{2}=\frac{2 m c^{2}}{\hbar^{2}}\left[\hbar \omega_{1}-\hbar \omega_{2}+E_{\sigma 0}-E_{\nu 0}\right]
$$

(36) and (35) are satisfied only in certain directions. Hence, in cases of collinear arrangement of wave vectors $\vec{k}_{1}$ and $\vec{k}_{2}$, the intensity of scattered radiation will be essentially dependent on the angles between $\vec{k}_{1}$ and optical axes. 
It should be noted that in the case when the energy of a "supracondensate "exciton is independent of the wave vector, i.e. $m=\infty$, the system of equations (32) has got the solution for any directions of incident and scattered radiation.

3. Vector synchronism. In this case, the solution of system (32) is of the form

$$
\sin ^{2} \frac{\theta}{2}=\frac{2 m c^{2} \hbar^{-1}}{\hbar}\left[\frac{\left(\omega_{1}+\omega_{\sigma}-\omega_{2}-\hbar^{-1} E_{v 0}\right)-\left(\omega_{1} n_{1}-\omega_{2} n_{2}\right)^{2}}{4 n_{1} n_{2} \omega_{1} \omega_{2}}\right]
$$

To study the nonlinear effect in cubic crystals, equation (37) was used for finding the scattering angles. Let the frequency of incident radiation be $\omega_{1}=3.66 \cdot 10^{15} \mathrm{sm}^{-1}$. Then, for crystals of ZnTe type (where $n=3.23 ; m=2.83 \cdot 10^{-28} \mathrm{~g}$; $\omega_{\sigma}=3.61 \cdot 10^{15} \mathrm{sm}^{-1}$ ), the following results are obtained (see Table 1).

Table 1. The dependence of the scattering angle of the frequency vector in synchronism.

\begin{tabular}{llll}
\hline$\theta$ (degree) & $\omega_{2} \cdot 10^{15} \mathrm{sm}^{-1}$ & $\theta$ (degree) & $\omega_{2} \cdot 10^{15} \mathrm{sm}^{-1}$ \\
\hline 24,08 & 3.66215 & 102.04 & 3.6615 \\
34,4 & 3.66210 & 154.8 & 3.6611 \\
49,3 & 3.6620 & 169.7 & 3.66105 \\
\hline
\end{tabular}

As it can be seen from the table, the scattering angle is highly dependent on frequency $\omega_{2}$, i.e. the scattered radiation is very much dispersed.

II. Condensate and supracondensate excitons are at identical branches.

The case of $m=\infty$ is excluded. If this requirement is satisfied, then, as it follows from the first equation in (32), $\omega_{1}=\omega_{2}$ and the line with the participation of condensate would coincide with the Rayleigh one. Suppose that $\partial E_{v} / \partial q>0$. Then the scattering spectrum will be similar to that in Figure 3. Analogously to it. 1, system (32) not always has got the solution in this case. The angles associated with the collinear phase synchronism with forward and backward scattering are determined from equations (35) and (36). In the right - hand parts of those equations, it is assumed that $\sigma=v$. When $\partial E_{v} / \partial q<0$, the spectrum is similar to the represented in Figure 4.

Let the case of "easy" excitons be considered. Then lines of $\omega_{1}$ and $\omega_{2}$ (Figure 3 and 4 ) will be very close. They could only be distinguished in the case when the frequency shift $\left(\omega_{1}-\omega_{2}\right)$ is higher than the instrument resolution $\Delta v$, i.e. when the inequality

$$
\hbar(2 \pi m c)^{-1}\left(n_{1}^{2} \omega_{1}^{2}+n_{2}^{2} \omega_{2}^{2}-2 n_{1} n_{2} \omega_{1} \omega_{2} \cos \theta\right)>\Delta v
$$

is satisfied. Here $\theta$ is the scattering angle. From (38) it follows that the most favorable conditions for the effect of nonlinear scattering with the participation of condensate are the backward geometry and low effective masses of a supracondensate exciton.

\section{Shape of a Scattered Radiation Line}

Above, only the frequency dependence of scattering tensors was considered. However, near the bands of exciton absorption, the question of the spectral shape of scattered radiation line becomes very important.

As it is known [36], within the interaction representation, time dependence of probability amplitudes in state $|n\rangle$ is determined by the formula

$$
i \hbar b_{n}(t)=\sum_{m} V_{n m} b_{m}(t) e^{i\left(E_{n}-E_{m}\right) \hbar^{-1} t}
$$

where $V_{n m}$ is a matrix element of perturbation operator; $t$ is time. Suppose that the first resonance situation is valid. Then the effect goes as follows: first, condensate exciton $E_{\sigma 0}$ and polariton $\hbar \omega_{1}$ are annihilated. Because of this fact, a mechanical exciton $E_{S_{1}}$ is created. After that it is decomposed to polariton $\hbar \omega_{2}$ and exciton $E_{s}$. In this case, the energy $\hbar \omega_{1}+E_{\sigma 0}$ coincides with that of mechanical exciton $E_{s_{1}}$. To solve (39), we use a Fourier transform

$$
b_{n}(t)=-\frac{1}{2 \pi i} \int_{-\infty}^{+\infty} d E G_{n 0}(E) \exp \left\{i\left(E_{n}-E\right) \hbar^{-1} t\right\}
$$

For convenience, the multiplier $G_{00}$ is separated, then

$$
G_{n 0}(E)=u_{n 0}(E) G_{00}(E) \zeta\left(E-E_{n}\right) \quad(n \neq 0)
$$

where

$$
\varsigma(E)=\frac{P}{E}-i \pi \delta(E)
$$

$P$ means that the corresponding expression is taken in the sense of the principal value. For function $u_{n 0}$, the relations resulting from substitution of (41) into (40) are valid:

$$
\begin{gathered}
u_{s_{1}, \sigma \rho_{1}}=H_{s_{1}, \sigma \rho_{1}}+\sum_{s \rho_{2}} H_{s_{1}, s \rho_{2}} u_{s \rho_{2} 0}(E) \varsigma\left(E-E_{s \rho_{2}}\right), \\
u_{s \rho_{2}, \sigma \rho_{1}}=H_{s \rho_{2}, s_{1}} u_{s_{1}, \sigma \rho_{1}}(E) \zeta\left(E-E_{s_{1}}\right)
\end{gathered}
$$

Substituting (44) into (43), we find

$$
u_{s \rho_{2}, \sigma \rho_{1}}(E)=H_{s_{1}, \sigma \rho_{1}}-\frac{i \hbar \gamma}{2} u_{s_{1}, \sigma \rho_{1}}(E) \varsigma\left(E-E_{S_{1}}\right)
$$

where

$$
\frac{\hbar}{2} \gamma(E)=i \sum_{s \rho_{2}}\left|H_{s \rho_{2}, s_{1}}\right|^{2} \varsigma\left(E-E_{s \rho_{2}}\right) .
$$


Multiplying (45) by $E-E_{s_{1}}$,

$$
u_{s_{1}, \sigma \rho_{1}}(E)=\frac{\left(E-E_{s_{1}}\right) H_{s_{1}, \sigma \rho_{1}}}{\left(E-E_{s_{1}}\right)+\frac{i \hbar}{2} \gamma(E)}
$$

and from (44)

$$
u_{s_{1}, \sigma \rho_{1}}(E)=\frac{H_{s \rho_{2}, s_{1}} H_{s_{1}, \sigma \rho_{1}}}{\left(E-E_{s_{1}}\right)+\frac{i \hbar}{2} \gamma(E)},
$$

Hence, for $t \rightarrow \infty$

$$
\begin{aligned}
b_{s_{1}}(\infty)=\frac{u_{s_{1}, \sigma \rho_{1}}\left(E_{s_{1}}\right)}{E_{s_{1}}-E_{\sigma \rho_{1}}+\frac{i}{2} \hbar \Gamma\left(E_{s_{1}}\right)} & (49) \\
\left|b_{s \rho_{2}}(\infty)\right|^{2}= & \frac{\left|H_{s \rho_{2}, s_{1}}\right|^{2}\left|H_{s_{1}, \sigma \rho_{1}}\right|^{2}}{\left[E_{s \rho_{2}}-E_{\sigma \rho_{1}}+\frac{i \hbar}{2} \Gamma\left(E_{s \rho_{2}}\right)\right]^{2}\left[E_{s \rho_{2}}-E_{s_{1}}+\frac{i \hbar}{2} \Gamma\left(E_{s \rho_{2}}\right)\right]^{2}}
\end{aligned}
$$

$$
b_{s \rho_{2}}(\infty)=\frac{u_{s \rho_{2}, \sigma \rho_{1}}\left(E_{s \rho_{2}}\right)}{E_{s \rho_{2}}-E_{\sigma \rho_{1}}+\frac{i}{2} \hbar \Gamma\left(E_{s \rho_{2}}\right)}
$$

Where

$$
\begin{aligned}
& E_{s \rho_{2}}=E_{s}+\hbar \omega_{1}, \quad E_{\sigma \rho_{1}}=E_{0 \sigma}+\hbar \omega_{1}, \\
& \frac{\hbar}{2} \Gamma(E)=i \sum_{s_{1} \rho_{1}} \frac{\left|H_{s_{1}, \sigma \rho_{1}}\right|^{2}}{E-E_{s_{1}}+\frac{i}{2} \hbar \gamma(E)}
\end{aligned}
$$

With (50) squared, the scattering probability is
As it follows from (43), for $E \sim E_{s_{1}}$, magnitude $\gamma$ is, in fact, a probability of decomposition of exciton $E_{S_{1}}$ into polariton $\hbar \omega_{2}$ and exciton $E_{s}$. It determines the radiation broadening of excitonic levels $s_{1}$. Magnitude $\Gamma(E)(51)$ is a broadening of $E_{\sigma \rho_{1}}$ state due to its finite lifetime for
$E=E_{\sigma \rho_{1}}$. It follows that the real and imaginary parts of $\Gamma$ and $\gamma$ are responsible for the broadening of the related levels and the shifts, respectively. The latter values are not of interest. Now, we replace $\gamma$ and $\Gamma$ with their real parts and denote them as $\gamma$ and $\Gamma$ once again. Then

$$
\begin{aligned}
& \gamma(E)=\frac{2 \pi}{\hbar} \sum_{s \rho_{2}}\left|H_{s \rho_{2}, s_{1}}\right|^{2} \delta\left(E-E_{s \rho_{2}}\right)=\frac{2 \pi}{\hbar} \int d \Omega_{\rho_{2}} d \Omega_{s}\left|H_{s \rho_{2}, s_{1}}\right|^{2} \rho\left(E_{s}\right) \rho\left(\hbar \omega_{2}\right), \\
& \Gamma(E)=\gamma \sum_{s, \rho_{1}} \frac{\left|H_{s_{1}, \sigma \rho_{1}}\right|^{2}\left(E-E_{s_{1}}\right)^{2}+\frac{\hbar^{2} \gamma^{2}}{4}}{}
\end{aligned}
$$

Here $\Omega_{n}$ is a solid angle with the emitted quantum $E_{n} ; \rho\left(E_{n}\right)$ is the density of states.

Now two cases are studied.

1. A crystal is exposed to broad - band radiation of intensity $I$ which is the constant in the region of radiation broadening of level $s$. As $I$ is constant, $\sum_{\rho_{1}}$ can be replaced by the integral. In the limit of $\gamma \rightarrow 0$, with only one levels taken into account, we have

$$
\Gamma=\frac{V}{(2 \pi)^{2}} \int d^{3} k_{1}\left|H_{s_{1}, \sigma \rho_{1}}\right|^{2} \delta\left(E_{\sigma \rho_{1}}-E_{s_{1}}\right)=\frac{2 \pi I V n_{0}}{\hbar^{2} \omega_{1} v_{1}}\left|H_{s_{1}, \sigma \rho_{1}}\right|^{2} \rho\left(\omega_{1}\right)
$$

where $\rho(\omega)$ is density of states; I is primary intensity; $\hbar \omega_{1}=E_{s_{1}}-E_{\sigma 0} ; v_{1}$ is the group velocity of polariton $\hbar \omega_{1}$. Expression (54) performs the total probability of polariton $\hbar \omega_{1}$ and condensate exciton $E_{\sigma 0}$ absorption per unit time. So, $\Gamma$ could be considered to be small enough. As follows from (52), for small $\Gamma$, the first multiplier of denominator is a $\delta$ - function. Thus, the difference $\left(E_{s \rho_{2}}-E_{\sigma \rho_{1}}\right)$ is different from zero only by $\hbar \Gamma$, i.e. the law of energy conservation is valid for scattering.

Consider now the shapes of absorption and scattering lines. For probability of emission, it follows from (52) and (54): 


$$
\sum_{\rho_{1}}\left|b_{s \rho_{1}}(\infty)\right|^{2}=\frac{\left|H_{s \rho_{2}, s_{1}}\right|^{2}}{\left(E_{s \rho_{2}}-E_{s_{1}}\right)^{2}+\frac{\hbar^{2} \gamma^{2}}{4}}
$$

The expression coincides with the shape of line for spontaneous irradiation. For absorption probability we have

$$
\sum_{\rho_{2}}\left|b_{s \rho_{2}}(\infty)\right|^{2}=\frac{\gamma}{\Gamma} \frac{\left|H_{s \rho_{2}, s_{1}}\right|^{2}}{\left(E_{s \rho_{2}}-E_{s_{1}}\right)^{2}+\frac{\hbar^{2} \gamma^{2}}{4}}
$$

As seen from (55), (56) the shapes of absorption and emission lines coincide. It can be concluded that when primary intensity is a broad - band and constant in the region of radiation broadening of the resonance level $E_{S_{1}}$, the process behaves in the same way as in the case of two independent processes, i.e. absorption and emission (as compared to the shape of the absorbed and emitted lines). However, the formula (52) for probability of the process does not coincide with the product of emission and absorption probabilities, since it does not connect energies $E_{\sigma \rho_{1}}$ and $E_{s_{1}}$ by a $\delta$ - function.

2. A crystal is exposed to narrow - band radiation, the linewidth being small as compared to radiation broadening level $s_{1}$. Then it can be supposed that the primary intensity is nonzero only at frequency $\omega_{1}$. The intensity of emitted line is obtained by making the summation (52) over $\rho_{1}$. In denominator, the first multiplier is a $\delta$ - function. Hence, $E_{s \rho_{2}}=E_{\sigma \rho_{1}}$. The integration gives

$$
\sum_{\rho_{1}}\left|b_{s \rho_{2}}(\infty)\right|^{2}=\frac{2 \pi I\left(\omega_{1}\right) n_{\sigma}\left|H_{s \rho_{2}, s_{1}}\right|^{2}\left|H_{s_{1}, \sigma \rho_{1}}\right|^{2} \rho(\omega)}{\hbar^{2} \Gamma v_{1}\left[\left(E_{s_{1}}-E_{\sigma \rho_{1}}\right)^{2}+\frac{\hbar^{2} \gamma^{2}}{4}\right]}
$$

It follows from (57) that as the denominator is constant in the area where $I(\omega)$ is nonzero, the shape of emission line is defined by the primary intensity. The emitted line should be of the same width as pumping. Thus, it can be concluded that during the excitation by a narrow spectral line, the scattering process should be considered as a single act, not two consecutive independent acts.

Some other resonant situations could be considered in this way. But in the case of conditions №2 and №4, in the intermediate state, a polariton wave originates and this fact should be taken into account. In those cases, the corresponding small denominator is provided by the choice of both the pumping frequency and the direction of radiation propagation. The value similar to $\gamma$ (46) will characterize the line shape for a wave originating in the intermediate state.

The calculations similar to the present ones confirm that if a narrow - band radiation is falling (incident) on the crystal and none of frequencies satisfies the conditions of synchronism, the process should be treated as a single one. If one of the frequencies satisfies the conditions of synchronism and pumping is broad - band and of constant intensity in the region of wave broadening in the intermediate state, the process should be treated as a cascade one.

\section{Conclusions}

After obtaining of exciton polariton condensates in the scientific community, an intense studies both theoretical and experimental aspects of this phenomenon are developed [3843]. Experimental observation of the considered four-body process in the crystal requires BEC of excitons to be formed. At the same time, conditions of collinear and phase synchronisms should be taken into account. As a consequence of theoretical group analysis of the scattering tensor, both dipole excitons that are able to interact with the light (polaritons) and dipole-less excitons can participate in afour-body process. Besides, detection of BEC in the state of $k=0$ becomes possible, contrary to the case of luminescence application. It should be noted that non-linear effects can be also applied to the existence proof of excitons BEC in onedimensional non-ideal array of coupled microcavities containingquantum dots [44] where the minima of the polaritons energy in the states of $k \neq 0$ are identified.

\section{References}

[1] A. Einstein, "Quantentheorie des Einatomigen idealen gases I". Sitzungsber. Preuss. Akad. Wiss., Phys. Math. K1, v. 22, pp. 261-267, 1924

[2] A. Einstein, "Quantentheorie des Einatomigen idealen gases II". Sitzungsber. Preuss. Akad. Wiss., Phys. Math. Kl, v. 1, pp. 3-14, 1925.

[3] M. N. Anderson, J. R. Ensher, M. R. Matthews, C. E. Wieman, E. A. Cornell, "Observation of Bose-Einstein Condensation in a Dilute Atomic Vapor", Science, v. 260, pp. 198-201, 1995.

[4] A. J. Legget, "Bose-Einstein condensation in the alkali-gases: Some fundamental concepts", Rev. Mod. Phys., v. 73, pp. 307-356, 2001.

[5] W. Ketterle. "Nobel lecture: When atoms behave as waves: Bose-Einstein condensation and the atom laser", Rev. Mod. Phys., v. 74, pp. 1131-1151, October 2002.

[6] William J. Mullin, Asaad R. Sakhel, "Generalized BoseEinstein Condensation", J. Low Temp. Phys., vol. 166, pp. 125-150, February 2012.

[7] S. A. Moskalenko, D. W. Snoke. Bose - Einstein condensation of excitons and biexcitons and coherent nonlinear optics with excitons. Cambridhe University Press, Cambridhe, 2000

[8] Tim Byrnes, Na Young Kim and Yoshihisa Yamamoto, "Exciton-polariton condensates", Nature Physics, v. 10, pp. 803-, 2014.

[9] Christopoulos, S. et al. "Room-temperature polaritonlasing in semiconductor microcavities". Phys. Rev. Lett. 98, 126405 (2007). 
[10] Baumberg, J. J. et al. "Spontaneous polarization buildupin a room-temperature polariton laser". Phys. Rev. Lett., v. 101, $136409,2008$.

[11] Kena-Cohen, S. k Forrest, S. R., "Room-temperaturepolariton lasing in an organic single-crystal microcavity", Nature Photonics, v. 4, pp. 371-375, 2010.

[12] Guillet, T. et al. "Polariton lasing in a hybrid bulk $\mathrm{ZnO}$ microcavity". Appl. Phys. Lett., v. 99, 161104, 2011.

[13] C. Liang, K. Wei, B. J. Ye, H. M. Wen, X. Y. Zhou, R. D. Han,"Green's function method to the ground state properties of a two-component Bose-Einstein condensate", Low Temperature Physics, v. 37, pp. 561- 567, July 2011.

[14] V. B. Timofeev, A. V. Gorbunov and D. A. Demin, "BoseEinstein condensation of dipolar excitons in lateral traps", Low Temperature Physics, v. 37, pp. 179-188, March 2011.

[15] C. T. Belyaev. "Application of the methods of quantum field theory to a system of Bose particles", Sov. Phys. JETP, v. 34, pp. 417-432, 1958.

[16] S. A. Moskalenko, M. A. Liberman, E. S. Moskalenko, E. V. Dumanov, I. V. Podlesny, "Coherence of two-dimensional electron-hole systems: Spontaneous breaking of continuous symmetries: A review", Physics of the Solid State, v. 55, pp. 1563-1595, August 2013.

[17] B. L. Halperin, P. A. Lee, N. Read. "Theory of the Half-filled Landau Level”, Phys. Rev. B, v. 47, pp, 7312- 7343, March 1993.

[18] L. V. Keldysh, Yu. V. Kopayev. "Possible instability of semimetallic state with respect to Coulomb interection", Physics of the Solid State, v. 6, pp. 2791-2798, 1964.

[19] G. Batyev, V. M. Kovalev, A. V. Chaplik, "Response of a Bose-Einstein condensate of dipole excitons to static and dynamic perturbations“", JETP Letters, v. 99, pp. 540-551, July 2014.

[20] Yu. D. Zavorotnev, O. Yu. Popova, "Polariton Spectrum Subject to Bose-Einstein Condensate of Excitons", Journal of Photonic Materials and Technology, v. 1, pp. 10-14, July 2015.

[21] Jesus Martinez-Linares, G. S. Agarwal. "Raman scattering from a Bose condensate", Phys. Rev. A, v. 57, pp. 2931-2937, April 1998.

[22] Yu. D. Zavorotnev, L. N. Ovander. "Raman scattering with participation of the Bose-Einstein condensate of excitons". Bulletin of the Russian Academy of Sciences: Physics, v. 69, pp. 984-987, July 2005.

[23] Yu. E. Lozovik, A. V. Pushnov, "New method for detection of exciton Bose condensation using stimulated two-photon emission”, JETP, vol. 88, pp. 747-760, April 1999.

[24] Yu. E. Lozovik, I. V. Ovchinnikov, "Stimulated multiphoton emission from exciton Bose condensate", JETP Letters, v. 75, pp. 507-512, May 2002.

[25] L. N. Ovander, "Nonlinear optical effects in crystals", Sov. Phys. Usp, v. 8, pp. 337-359, August 1965.

[26] Yu. D. Zavorotnev, L. N. Ovander. “К теории ГКР”, Physics of the Solid State, v. 16, pp. 2387-2392, 1974.

[27] N. Bloembergen. Nonlinear optics/ 4th edition. Word Scientific Publishing Co, London, 1996. 173p.
[28] V. M. Agranovich, "Effects of the internal field in the spectra of molecular crystals and the theory of excitons", Sov. Phys. Usp., v. 17, pp. 103-117, 1974.

[29] V. M. Agranovich, "Effects of the internal field in the spectra of molecular crystals and the theory of excitons", Sov. Phys. Usp., v. 17, pp. 103-117, 1974.

[30] V. M. Agranovich, Theory of Excitons, Nauka Publishers, Moscow, 1968. 328 p.

[31] D. I. Lalovic, B. S. Tosic, R. R. Zakula. "Theory of Nonlinear Effects in Crystals in the Second-Quantization Representation", Phys. Rev. v. 178, pp. 1472-1479, February 1969.

[32] P. A. Apanasevich. Bases of the theory of interaction of light with matter. Science and Technique Publishers, Minsk, 1977. 496 p.[in Russian].

[33] V. V. Lavric, L. N. Ovander, V. T. Shunyakov. "Tensor of the nonlinear polarizability of anisotropic medium and "local" field method", Sol. State Commun, v. 47, pp. 355-359, 1983.

[34] L. D. Landau, E. M. Lifshitz. Quantum Mechanics. Pergamon Press, Oxford, 1974. 702 p.

[35] L. N. Ovander. "On the form of the Raman scattering tensor", Optics and Spectroscopy, v. 9, pp. 571- 575, 1960.

[36] L. G. Koreneva, V. F. Zolin, B. L. Davydov. Molerular crystals in nonlinear optics. Nauka Publishers, Moscow. 1975. 138 p. [in Russian].

[37] W. Heitler. The Quantum Theory of Radiation. 3d Edition. Dover Publishers, NY, 1954. 430 p.

[38] Konstantinos Lagoudakis the Physics of Exciton-Polariton Condensates. EPEL Press, Lausanne, Switzerland, 2013. $164 \mathrm{p}$.

[39] Benoit Deveaud. "Exciton-Polariton Bose-Einstein Condensates", Annual Review of Condensed Matter Physics, v. 6:155-175, March 2015.

[40] Nataliya Bobrovska, Michał Matuszewski, Konstantinos S. Daskalakis, Stefan A. Maier, and Stéphane Kéna-Cohen. "Dynamical Instability of a Nonequilibrium ExcitonPolariton, Condensate" ACS Photonics, v. 5 (1), pp. 111-118, 2018.

[41] Saeed A Khan and Hakan E Türeci. "Competing role of interactions in synchronisation of exciton-polariton condensates", New Journal of Physics, v. 19:105008, October 2017.

[42] Tomoyuki Horikiri, Tim Byrnes, Kenichiro Kusudo, Natsuko Ishida, Yasuhiro Matsuo, Yutaka Shikano, Andreas Löffler, Sven Höfling, Alfred Forchel, and Yoshihisa Yamamoto, "Highly excited exciton-polariton condensates", Phys. Rev. B 95, 245122, 19 June 2017.

[43] Fabio Scafirimuto, Darius Urbonas, Ullrich Scherf, Rainer F. Mahrt, and Thilo Stöferle "Room-Temperature ExcitonPolariton Condensation in a Tunable Zero-Dimensional Microcavity", ACS Photonics, v. 5 (1), pp. 85-89, 2018, Web: September 29, 201.

[44] V. V. Rumyantsev, S. A. Fedorov, and K. V. Gumennik. "Polariton Excitations in a Non-Ideal Array of Microcavities with Quantum Dots", Physics of the Solid State, Vol. 59, No. 4, pp. 758-765, 2017. 